\title{
Evaluation of Data Collection Capability in a Large-Scale Smart Metering System Based on Receiver-Driven Multihop Communication
}

\author{
Masashi Sugano, Taichi Shimizu, and Naoki Wakamiya
}

\begin{abstract}
We consider a large-scale smart metering system based on intermittent receiver driven transmission and potential-based routing, both of which have been developed for wireless sensor networks. In this paper, we evaluate the performance of such a system by simulations and show that it is possible to collect $99 \%$ of data within 10 minutes in a system of 1600 nodes.
\end{abstract}

Index Terms-Multihop communication, potential routing, smart meter, wireless communication

\section{INTRODUCTION}

In order to satisfy the growing energy demand of recent years, interest is growing in smart grids, which enable efficient energy use by utilizing information and communication technology [1]. Fig. 1 shows the concept of a smart metering system, which consists of hundreds of sets of smart meters and one center station (sink node). Each meter measures the electricity usage for a definite period of time (e.g., 30 minutes), and transmits the data upstream to the sink node. In addition, it is necessary for there to be downstream transmission to meters from the sink node for purposes such as power transmission control and maintenance (Fig. 1). Therefore, the communication method used for a smart metering system needs to provide two-way communication. Moreover, in order to monitor real time power consumption, it is also necessary to shorten the time required for data collection [2].

As a method for realizing such a large-scale network, we have considered using IRDT (Intermittent Receiver Driven Transmission) [3,4] for building a receiver-driven wireless multihop network. IRDT is suitable for networks, like a mesh network, that are densely deployed and in which data can take multiple routes. A routing protocol based on a routing table is used in the original IRDT design. However, this method lacks scalability and is not suitable for a large network. In contrast, potential based routing, which is used in wireless mesh networks, possesses excellent scalability $[5,6]$. So we have

Manuscript received June 25, 2013; revised August 30, 2013. This work was supported in part by "Grant-in-Aid for Scientific Research (C) 23500097" of Japan Society for the Promotion of Science (JSPS) in Japan.

Masashi Sugano is with the School of Knowledge and Information System, Osaka Prefecture University, Habikino-shi, 5838555 Osaka, Japan (e-mail: sugano@kis.osakafu-u.ac.jp).

Taichi Shimizu was with the Department of Information and Computer Sciences, Osaka University, Toyonaka-shi, 5608531 Osaka, Japan (e-mail: taichi-s@ics.es.osaka-u.ac.jp).

Naoki Wakamiya is with the Graduate School of Information Science and Technology, Osaka University, Suita-shi, 5650871 Osaka, Japan (e-mail: wakamiya@ist.osaka-u.ac.jp). proposed combining IRDT with the potential based routing [7] and have subsequently shown that it is possible to realize downstream communication [8]. Therefore, this combination may be able to provide the performance demanded of a smart metering system.

Until now, only the performance of a comparatively small-scale IRDT network of about 50 nodes has been evaluated; it has not been clearly shown what kind of performance is obtained for a large-scale IRDT network. In this paper, we consider a large-scale smart metering system based on IRDT combined with potential based routing and evaluate its performance by simulation experiments. In addition, we identify a problem and propose a technique for improving performance.

We begin by introducing IRDT and potential based routing in Section II. In Section III we identify a problem with applying the methods to a large-scale smart metering system, and propose a technique for performance improvement. In Section IV we evaluate the performance of the proposed method, and in Section V we present our conclusions and describe future work in.

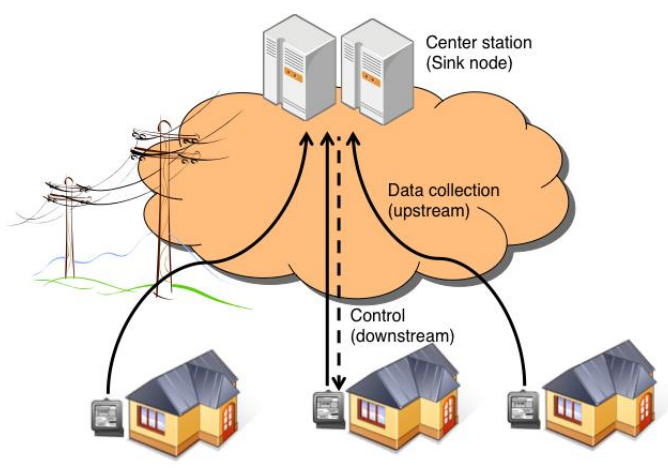

Fig. 1. Conceptual diagram of a smart metering system.

\section{OVERVIEW OF IRDT}

\section{A. MAC Layer Protocol}

In the IRDT protocol, each node intermittently wakes and transmits its ID to adjacent nodes in a very short packet. If no reply is received after a short time, the node sleeps again. Nodes holding transmission data wait for an ID packet from suitable destination nodes, and upon receiving one, respond with a send request (SREQ) packet. After receiving a relay acknowledgement (RACK) packet, the sender transmits its data packet and ends communication following receipt of a data acknowledgement (DACK) packet. Fig. 2 shows this intermittent operation involving nodes labeled Receiver 1 and Receiver 2. Here, the sender node accepts the ID of 
Receiver 2 as an appropriate receiver, as determined by the routing protocol. Having many nodes that are suitable destinations shortens the waiting time for ID packets, reduces power consumption, and improves communication reliability.

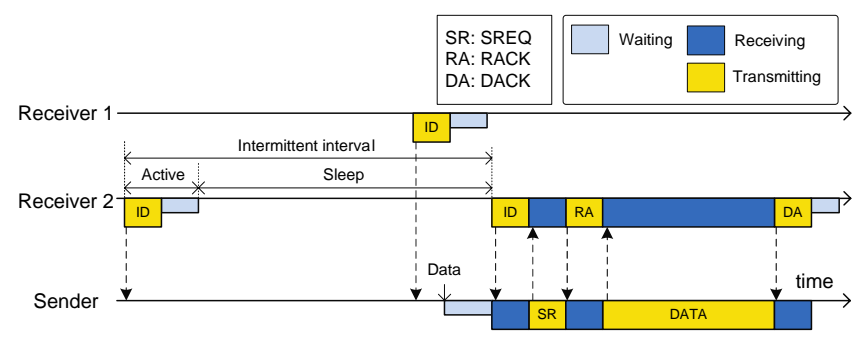

Fig. 2. Data transmission process of the MAC protocol of IRDT.

\section{B. Routing Protocol}

In IRDT, each node has a relay function and transmits data by multi-hop communication. In order to reduce transmission delays, it is necessary to transmit a packet along a route in which the number of hops to the sink is as small as possible. However, since the quality of wireless communication can be greatly affected by the environment, a routing method which can respond flexibly to such changes is desirable. In potential routing, all the nodes have a scalar value called the potential, and local routing is based on relative sizes of this value. So routing can respond flexibly to change by controlling potential values based on environment, load, etc.

Each time a node transmits an ID packet, it includes its own potential value. When a sender node receives the ID from a receiver node, it compares its own potential value with the potential value included in the ID. If the receiver is nearer to a sink, the potential value of a receiver will be larger than that of the sender and transmission is performed. Nodes which are within communication range of a particular are called adjacent nodes. Adjacent nodes with a larger potential value are called "forward nodes", those with a comparable potential value are "sideward nodes", and nodes with a smaller potential value are "backward nodes" (Fig. 3). Although a node's potential value should be determined based on the potentials of adjacent nodes and number of hops from the sink node, we specified potential values based on the number of hop beforehand for convenience. Therefore, we do not consider potential change in this paper.

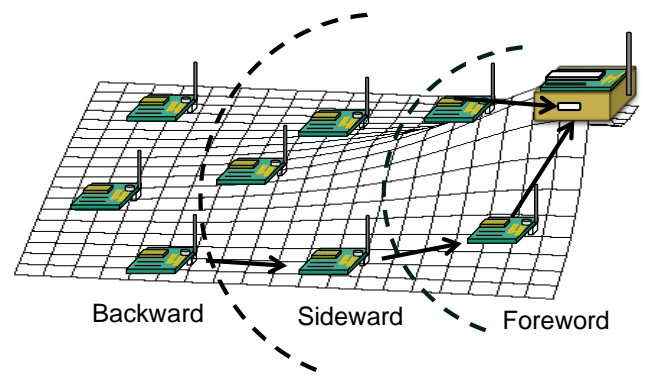

Fig. 3. Classification of adjacent nodes by the difference in its potential value.

\section{ApPlying IRDT to a LARGe-Scale SMARt Metering SYSTEM}

\section{A. Simulation Settings}

For the simulation experiments we used OMNeT++ [9] which was developed for the simulation of a large-scale mesh network. The simulated system is a smart metering system of 500 nodes placed on a grid at intervals of $17 \mathrm{~m}$ in a $400 \mathrm{~m} \mathrm{x}$ $400 \mathrm{~m}$ region, with one center station (sink node) placed at the center of the region. In this research, for simplicity, potential value is determined only by the number of hops from the sink node and the assigned values are shown in Fig. 4.

We performed the simulation with settings shown in Table I. The timeout is the maximum time that a node can retain a data packet. If transmission of a data packet fails, a node will try to send it again if the elapsed time is less than the timeout; but if the elapsed time exceeds the timeout, it will discard the packet. TTL (Time to Live) is the maximum transmission frequency of a data packet. When each node relays a data packet, it decrements this value, and a packet is discarded when this value reaches 0 . In this environment, we investigated the packet collection rate and delay based on making 45 transmissions at $500 \mathrm{~s}$ intervals.

\section{B. Problem of IRDT}

Fig. 5 shows distribution of the number of packets which each node discarded in the simulation described above. All discards were due to timeouts; that is, a node has been unable to transmit a packet to a forward node for an extended period of time, and so the packet is discarded. Furthermore, as can be seen from Fig. 6, the nodes that discarded many packets are those that are most distant from the sink among nodes with the same potential value. These nodes have few forward nodes and many backward nodes. Therefore, they receive many packets from their backward nodes but, since there are few forward nodes, they may repeatedly fail to transmit (because none of the IDs received belong to a forward node) and eventually timeout, at which point these packets are discarded.

TABLE I: SIMULATION SETTINGS

\begin{tabular}{lr}
\hline Number of nodes & 500 \\
\hline Transmission range & $56 \mathrm{~m}$ \\
\hline Node placement interval & $17 \mathrm{~m}$ \\
\hline Transmitting packets & 45 \\
\hline Packet generation interval & $500 \mathrm{~s}$ \\
\hline Timeout & $100 \mathrm{~s}$ \\
\hline TTL (Time to live) & 15 \\
\hline Intermittence interval & $1 \mathrm{~s}$ \\
\hline
\end{tabular}

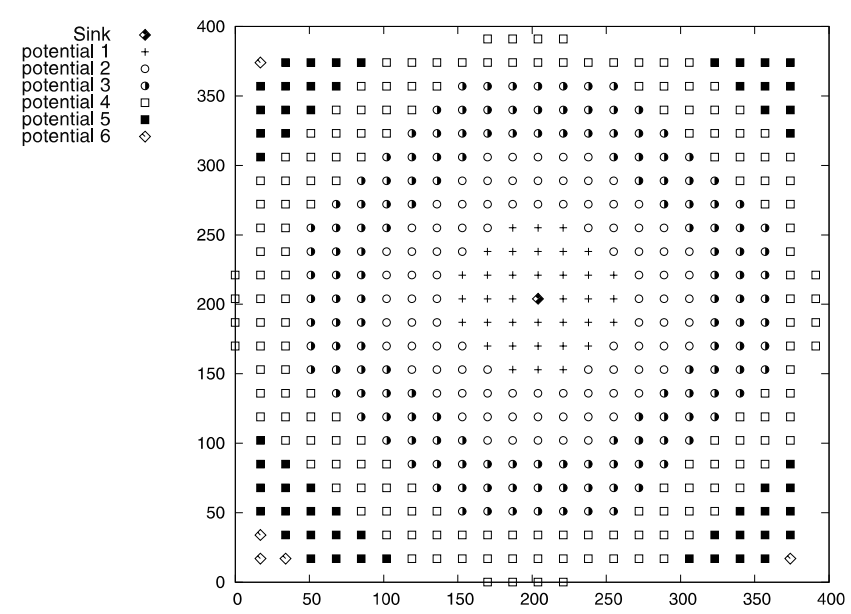

Fig. 4. Distribution of potential values (500 nodes, transmission range: 56 $\mathrm{m})$. 


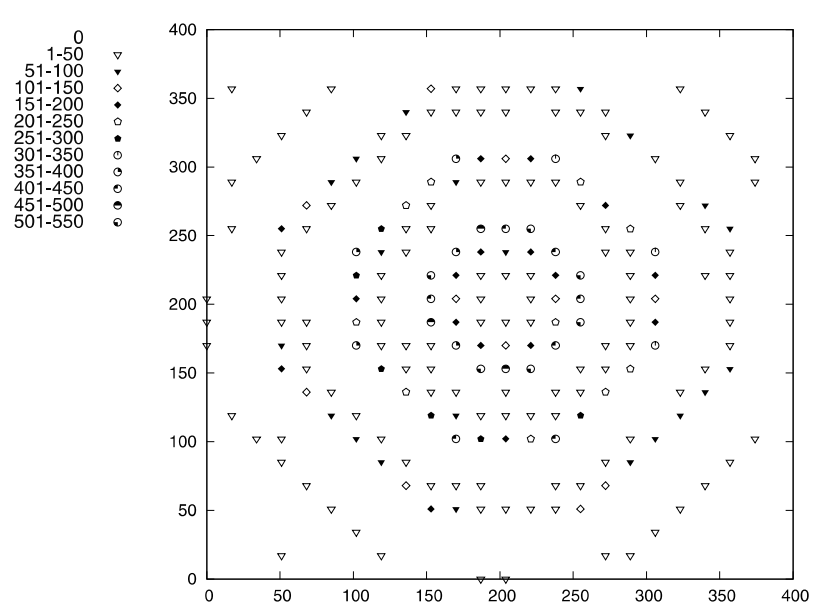

Fig. 5. Distribution of the number of packets discarded at each node.

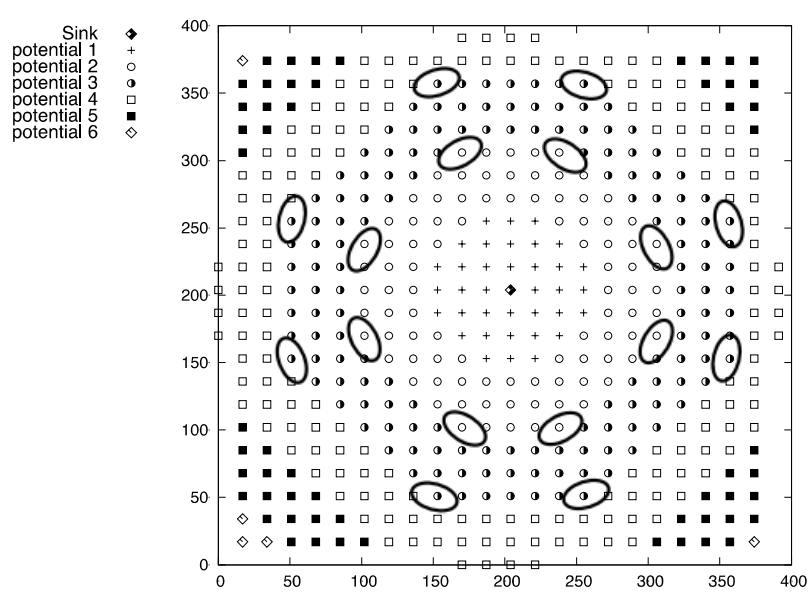

Fig. 6. The nodes which are most separated from the sink node in the node groups with potential values of 2 or 3 .

\section{Introducing Sideward Transmission}

If potential based routing is applied to IRDT in the simplest way, each node will transmit only to forward nodes (which have a smaller potential value) and IDs from sideward or backward nodes will be ignored. This leads to the problem of many packets being discarded at particular nodes. To solve this problem we allow transmission to a sideward node with probability $p$ [10]. That is, when an ID packet is received from a sideward node, a node responds with the probability $p$. All the nodes attempt transmission to a forward node first. After that, transmission to a sideward node is attempted with a probability that increases as the number of failed transmissions increases. For this study we defined the relation between the probability of sideward transmission, $p$ $(\%)$, and the number of failures $x$ as

$$
p=100^{\frac{x}{10}}
$$

The aim of allowing sideward transmission is to avoid packets being trapped at nodes with few forward nodes. However, if sideward transmission is successful, the packet has not moved any closer to a sink node. Therefore, we would like to keep sideward transmission to a necessary minimum. We achieve a balance between these two aims by giving the probability $p$ a small value initially and increasing it exponentially with the number of transmission failures. By applying this technique we were able to increase the packet collection rate, as shown in Table II. Moreover, as shown in Fig. 7, the impact of sideward transmission is that almost all packets are collectable within 3 minutes, although average delay is increased.

TABLE II: INCREASE IN PACKET COLLECTION RATE BY INTRODUCTION OF SIDEWARD TRANSMISSION

\begin{tabular}{lc}
\hline \multicolumn{2}{c}{ SIDEWARD TRANSMISSION } \\
\hline Without sideward transmission & Packet collection rate \\
\hline With sideward transmission & $93.0 \%$ \\
\hline
\end{tabular}

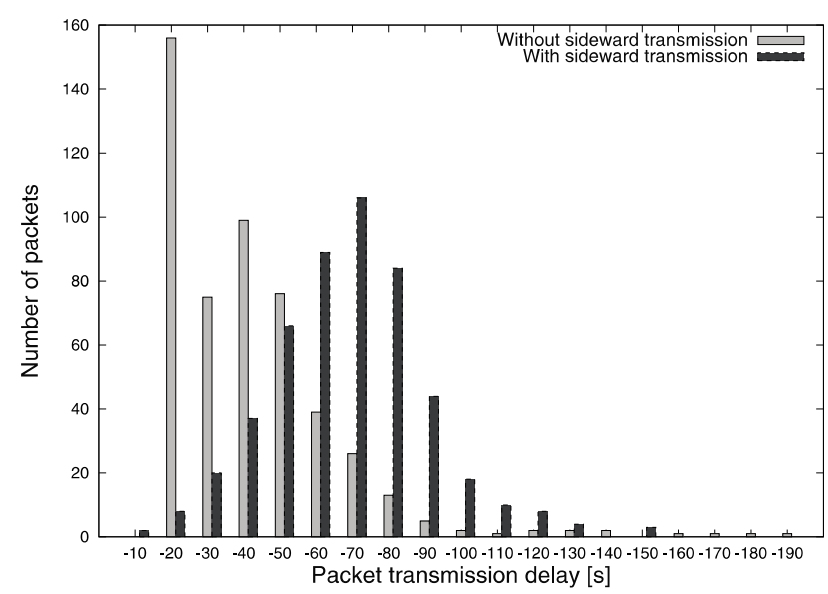

Fig. 7. Change of the delay distribution by the introduction of sideward transmission.

\section{Performance Evaluation}

\section{A. Derivation of the Optimal Intermittence Interval for the Sink Node}

The cause of the fall in the packet collection rate is the existence of nodes with fewer forward nodes than backward nodes. This is a particular problem for nodes which are adjacent to the sink node because, for these nodes, only the sink node is a forward node. Such a situation occurs for any system, like a sensor network, which collects data in a single sink. In this case, system-wide performance may be improved by increasing the throughput of the last hop. In IRDT, all the nodes, including the sink node, operate intermittently, and performance is affected by the intermittence interval. When the intermittence interval of the sink node is decreased, the amount of traffic per unit time increased, and we expected that more packets would be collectable. So we changed the intermittence interval of the sink node and investigated the impact which it has on the packet collection rate in the simulation.

In a network of 1024 nodes, we changed the intermittence interval of the sink node from $0.1 \mathrm{~s}$ to $0.0001 \mathrm{~s}$, and compared the packet collection rate for transmission ranges of $40 \mathrm{~m}, 56$ $\mathrm{m}, 72 \mathrm{~m}$, and $95 \mathrm{~m}$. Fig. 8 shows that the packet collection rate can be increased by making the intermittence interval of the sink smaller. However, if the intermittence interval is made too small too, the packet collection rate will decrease. This decrease is the result of interference caused to communications of other nodes within the communication range by the sink transmitting its ID packet at very short intervals. Thus, we judged the most suitable intermittence 
interval of the sink to be $0.001 \mathrm{~s}$ in our system.

Fixing the intermittence interval of the sink at $0.001 \mathrm{~s}$, we next investigated the packet collection rate for different numbers of nodes. From Fig. 9 it is clear that the best packet collection rate can be obtained for a communication range of $56 \mathrm{~m}$, independent of the size of the network. This result suggests that the packet collection rate in IRDT depends on the number of adjacent nodes of each node. From Table III it can be seen that the optimal communication range is such that the number of adjacent nodes is about 36 .

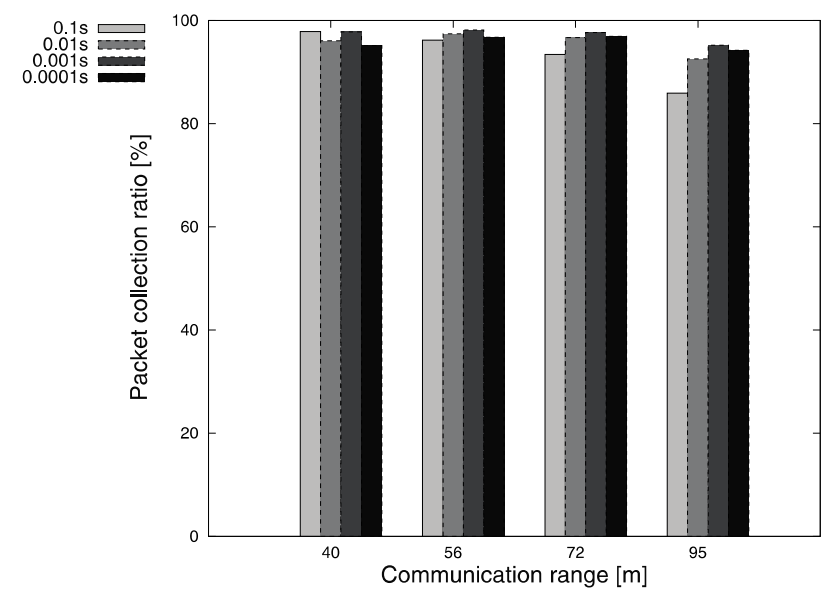

Fig. 8. Change of the data collection ratio with the operation interval of the sink.

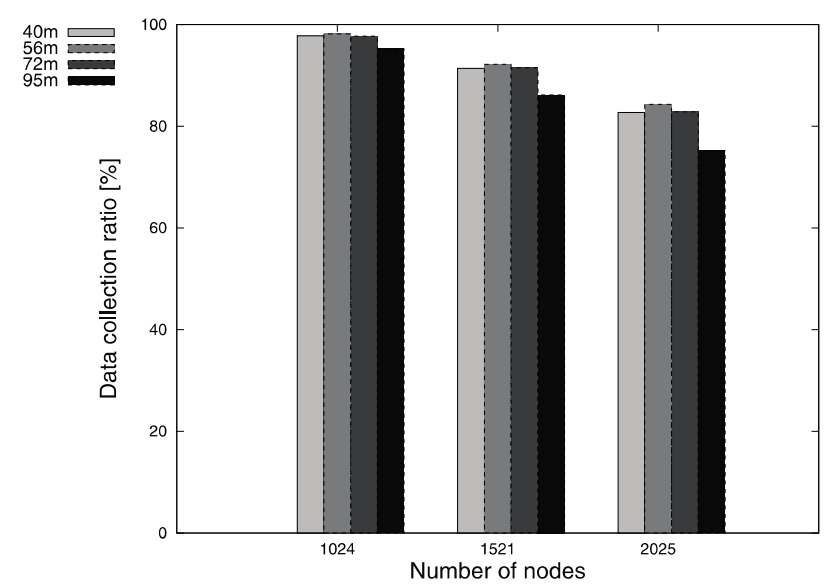

Fig. 9. Change of the packet collection rate for different transmission ranges.

TABLE III: THE ARRANGEMENT OF CHANNELS

\begin{tabular}{|c|c|}
\hline Transmission range $(\mathrm{m})$ & Number of adjacent nodes \\
\hline 40 & 20 \\
\hline 56 & 36 \\
\hline 72 & 56 \\
\hline 95 & 100 \\
\hline
\end{tabular}

\section{B. Improvement in Packet Collection Rate by Retransmission}

Packets which have not reached the sink after a certain amount of time has passed since the start of communication may have been trapped by a repeated transmission failure somewhere in the network. Since these packets are likely to be discarded as a result of timeouts, we discard all the packets that remain in the network at a fixed time after the start of communication. The packet collection rate could, therefore, be improved by transmitting again only those packets which have not reached the sink. (Because these packets are relatively few in number, the network load is light and there is less chance of a packet becoming trapped.) In order to realize such a method, it is necessary to send the demand for retransmission from the sink to the relevant nodes. However, in this study, we decided, for simplicity, to derive a collection rate by sending the request virtually so that the time taken for that request is zero.

In the final simulation, we interrupted transmission $300 \mathrm{~s}$ after the start of communication and discarded all the packets that had not reached a sink at that time. Then, we regenerated packets at those nodes whose original packets had failed to reach the sink and allowed transmission to take place for another $300 \mathrm{~s}$. We measured the packet collection rate $600 \mathrm{~s}$ after the start of communication for various sizes of network. Fig. 10 shows that we were able to achieve a packet collection rate of $99.9 \%$, when the number of nodes was 1500 . Even when the number of nodes was $2000,90.6 \%$ of packets were collected.

\section{CONCLUSION AND FUtURE WORK}

In this paper, we have proposed for technique for using IRDT in a large-scale smart metering system, and evaluated its performance by simulation. We found that there is an optimal transmission range that maximizes the packet collection rate, and that this range is not dependent on network scale. Moreover, it became possible to achieve a packet collection rate approaching $100 \%$ in 10 minutes for 1600-node networks by retransmitting packets which had not arrived at the sink after a fixed time.

As a subject of future research, the method for sending the retransmission request to each node from the sink needs to be investigated. For the result which we obtained in this study the retransmission request was performed virtually rather than by simulating the request using two-way IRDT communication. In a more realistic simulation, it is expected that the packet collection rate will be lower than the result shown here. Moreover, in this study, the nodes are laid out in a grid, and conditions prevailing in actual networks, such as the variation of wireless communication quality and varying communication distances in different directions, are not taken into account. It is necessary to evaluate networks in which these realistic conditions are present.

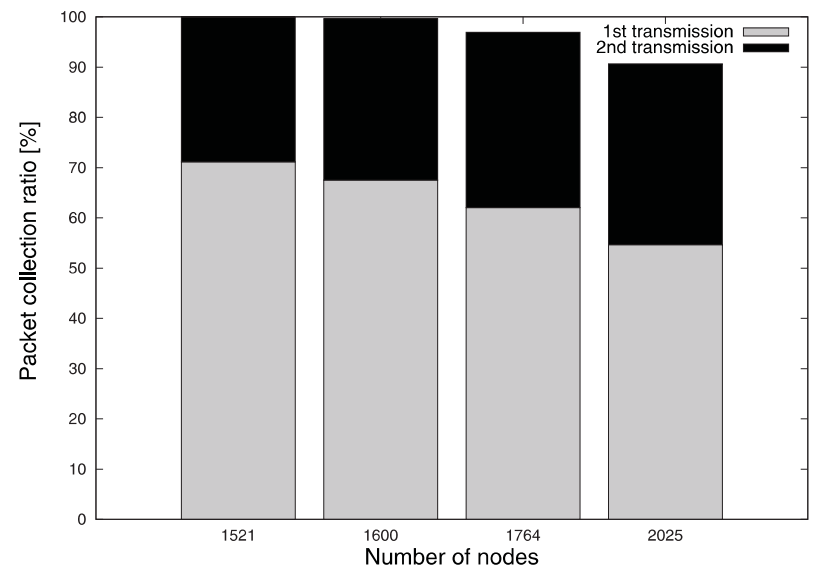

Fig. 10. Packet collection rates with and without retransmission for various network sizes. 


\section{REFERENCES}

[1] Z. Fan, G. Kalogridis, C. Efthymiou, M. Sooriyabandara, M. Serizawa, and J. McGeehan, "The new frontier of communications research: Smart grid and smart metering," in Proc. 1st International Conference on Energy-Efficient Computing and Networking, pp. 115-118, Aug. 2010.

[2] T. Song, D. Kaleshi, R. Zhou, O. Boudeville, J.-X. Ma, A. Pelletier, and I. Haddadi, "Performance evaluation of integrated smart energy soluations through large-scale simulations," in Proc. of 2nd IEEE International Conference on Smart Grid Communications (SmartGridComm 2011), pp. 37-42, Oct. 2011.

[3] D. Kominami, M. Sugano, M. Murata, T. Hatauchi, and J. Machida, "Energy-efficient receiver-driven wireless mesh sensor networks," Sensors, vol. 11, issue 1, pp. 111-137, Jan. 2011.

[4] IEEE Standard for Local and metropolitan area networks-Part 15.4: Low-Rate Wireless Personal Area Networks (LR-WPANs) Amendment 1: MAC sublayer, IEEE, IEEE Std 802.15.4e-2012 (Amendment to IEEE Std 802.15.4-2011), pp. 1-225, Feb. 2012.

[5] S. Jung, M. Kserawi, D. Lee, and J.-K. K. Rhee, "Distributed potential field based routing and autonomous load balancing for wireless mesh networks," IEEE Communications Letters, vol. 13, no. 6, pp. 429-431, Jun. 2009.

[6] C. Wu, R. Yuan, and H. Zhou, "A novel load balanced and lifetime maximization routing protocol in wireless sensor networks," in Proc. of IEEE VTC Spring 2008, pp.113-117, May 2008.

[7] D. Kominami, M. Sugano, M. Murata, and T. Hatauchi, "Controlled potential-based routing for large-scale wireless sensor networks," in Proc. of ACM MSWiM 2011, pp.187-195, Oct. 2011.

[8] S. Toyonaga, D. Kominami, M. Sugano, and M. Murata, "Potential-based downstream routing for wireless sensor networks," in Proc. of 7th International Conference on Systems and Networks Communications (ICSNC 2012), pp. 59-64, Nov. 2012.

[9] OMNeT++ Communit. OMNeT++ Network Simulation Framework. [Online]. Available: http://www.omnetpp.org/.

[10] D. Chuluunsuren, D. Kominami, M. Sugano, M. Murata, and T. Hatauchi, "Load balancing techniques for extending smart metering system lifetime," in Proc. 2012 IEEE Region 10 Conference (TENCON 2012), pp. 1-6, Nov. 2012.

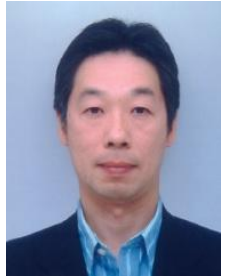

Masashi Sugano received the M.E. and D.E. degrees in Information and Computer Science from Osaka University, Japan, in 1988 and 1993, respectively. In April 1988, he joined Mita Industrial Co., Ltd. (currently, Kyocera Document Solutions Inc.) as a Researcher. From 1996 to 2003, he was an Associate Professor in Osaka Prefecture College of Health Sciences. From 2003 to 2005, he was an Associate Professor with the Faculty of Comprehensive Rehabilitation, Osaka Prefecture College of Nursing. From 2005 to 2012, he was with the School of Comprehensive Rehabilitation, Osaka Prefecture University, and From April 2009, he has been a Professor. He moved to School of Knowledge and Information Systems, College of Sustainable System Sciences, Osaka Prefecture University in April 2012. His current research interests include performance evaluation of computer communication network, network reliability, and ad hoc and sensor network systems. Prof. Sugano is a member of IEEE, ACM, IEICE, and IPSJ.

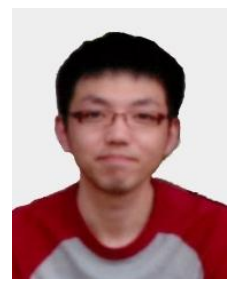

Taichi Shimizu graduated from School of Engineering Science, Osaka University, Japan in 2013. His work is on combination of a receiver-driven MAC protocol and a potential-based routing protocol for data collection in smart metering services.

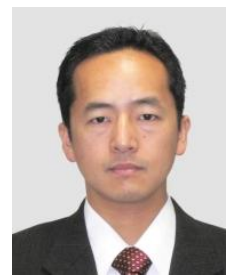

Naoki Wakamiya received M.E. and Ph.D. from Osaka University, Japan, in 1994 and 1996, respectively. Since then he has been with Osaka University as a faculty member and now he is a Professor of Graduate School of Information Science and Technology, Osaka University, since July 2011 His research interests include bio-ICT (biologicallyinspired information and communication technology) and self-organizing network control. He received the 2nd IEEE ComSoc Asia-Pacific Young Researcher Award in 2005. Prof. Wakamiya is a senior member of IEICE and a member of IPSJ, ACM, and IEEE. 